\title{
SUPPLEMENTARY ONLINE MATERIAL
}

Circadian clock properties of fruit flies Drosophila melanogaster exhibiting early and late emergence chronotypes

KL Nikhila, Koustubh M Vaze ${ }^{a, b}$, Karatgi Ratna and Vijay Kumar Sharma ${ }^{a *}$

${ }^{a}$ Chronobiology Laboratory, Evolutionary and Organismal Biology Unit, Jawaharlal Nehru Centre for Advanced Scientific Research, Jakkur, PO Box. 6436, Bangalore-560064, Karnataka, India

bPresent address: Neurobiology and Genetics, Theodor-Boveri Institute, Biocenter, University of Würzburg, Würzburg, Germany

Running Title: Chronotypes and circadian clocks.

${ }^{*}$ Correspondence to: Vijay Kumar Sharma E-mail: vsharma@jncasr.ac.in/vksharmas@gmail.com; Phone: +91-080-22082843 (office)/ 2844/45 (lab); Fax: +91-080-22081766; Url: http://www.jncasr.ac.in/vsharma. 


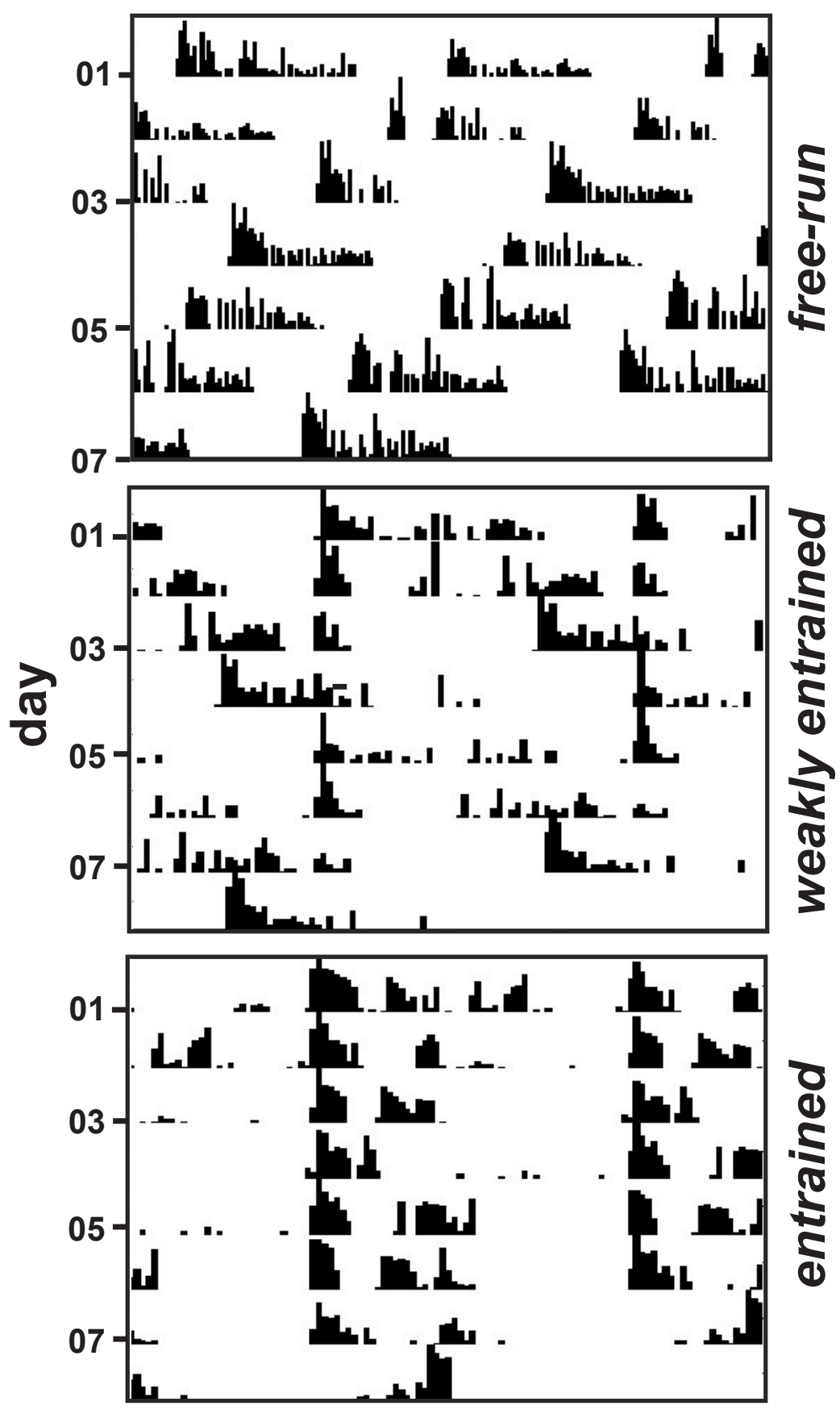

Supplementary Figure S1: Representative actograms of flies that exhibit free-run (neither morning nor evening activity components phaselocked to the LD cycle) in T30, weakly entrained (one of the two components phase-locked to the LD cycles while the other exhibited free-run) in T18, and entrained (both activity components phase-locked to the LD cycle) rhythm in $T 18$. 
(a)

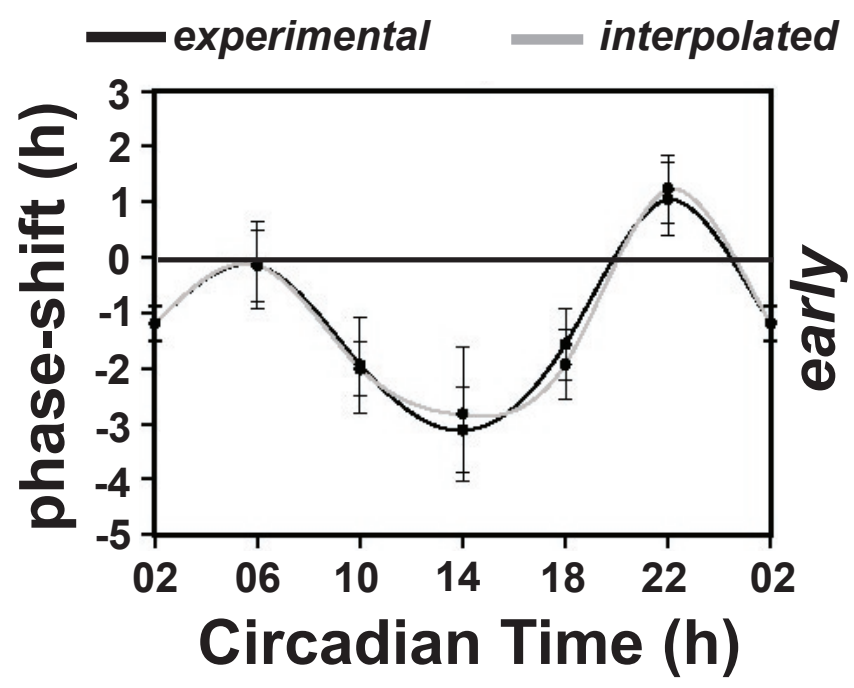

(c)

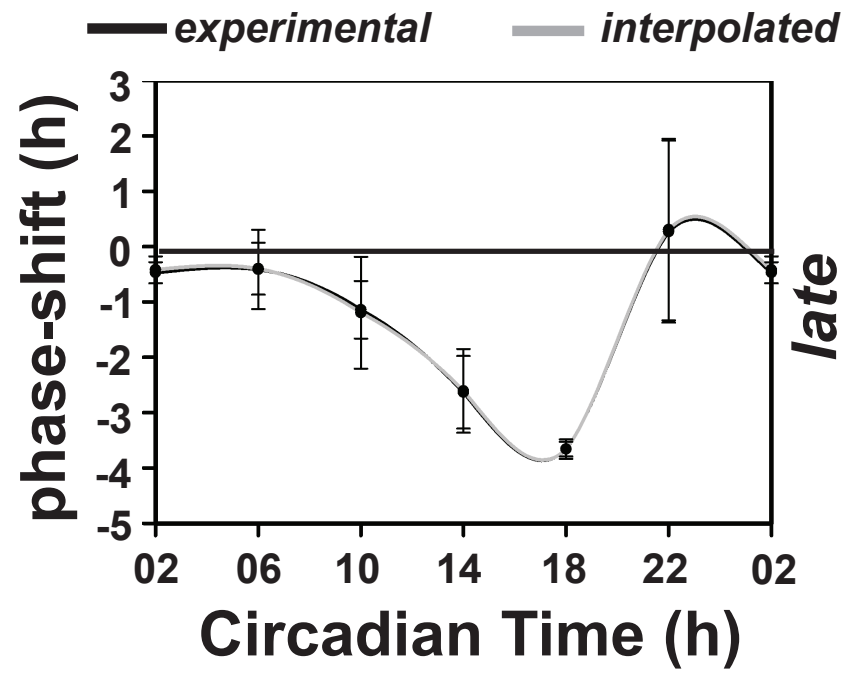

(b)

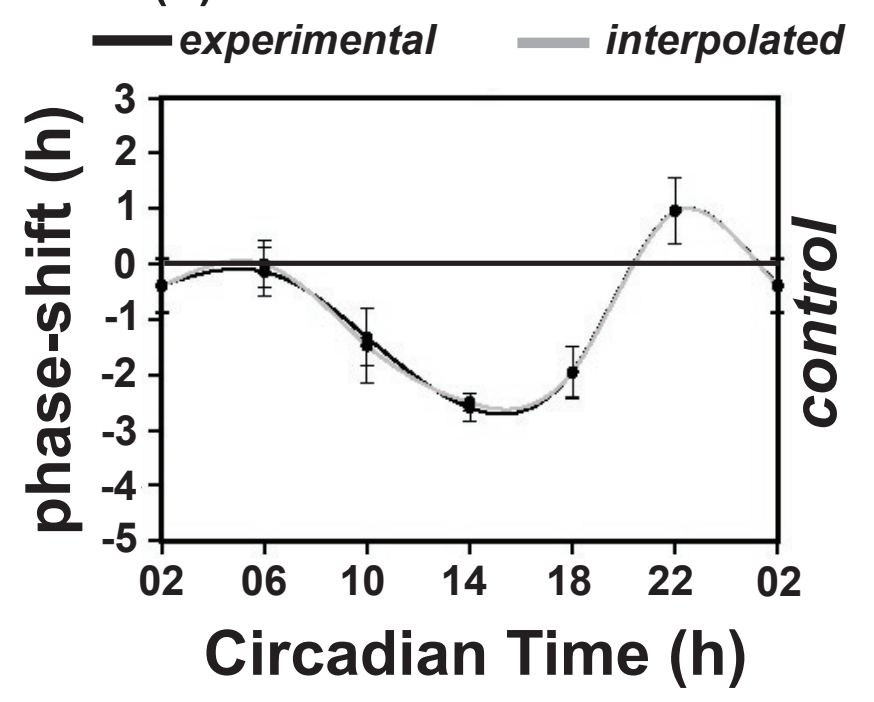

(d)

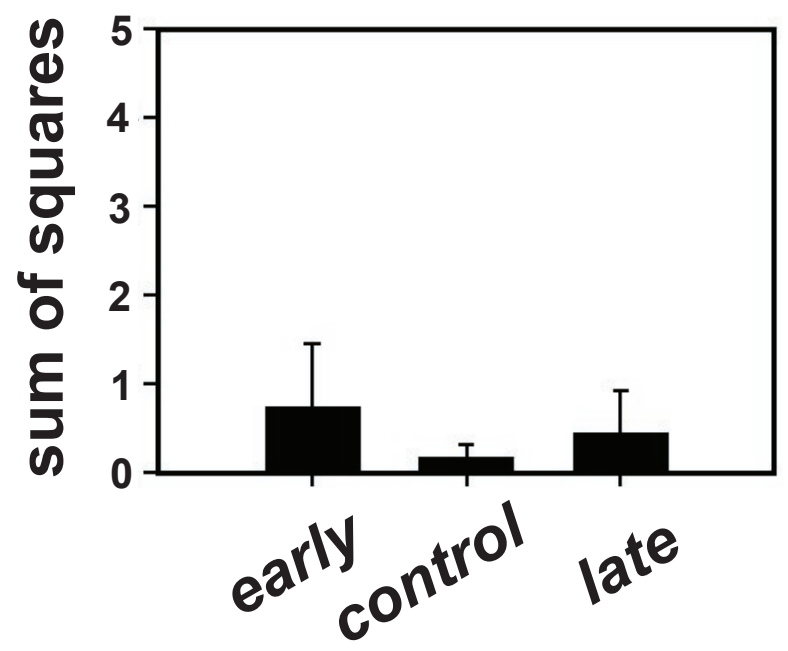

Supplementary Figure S2: Figures (a)-(c) represent the PRCs plotted from experimental (black line) and interpolated (grey line) data from polynomials used to etimate area under the curve for the (a) early (b) control and (c) late populations. (d) Sum of squares values indicating the goodness-of-fit between the polynomial and experimental data. All sum of square values reported here did not differ significantly from 0 thus indicating the polynomials used for AUC analysis reflects the experimental data accurately. 


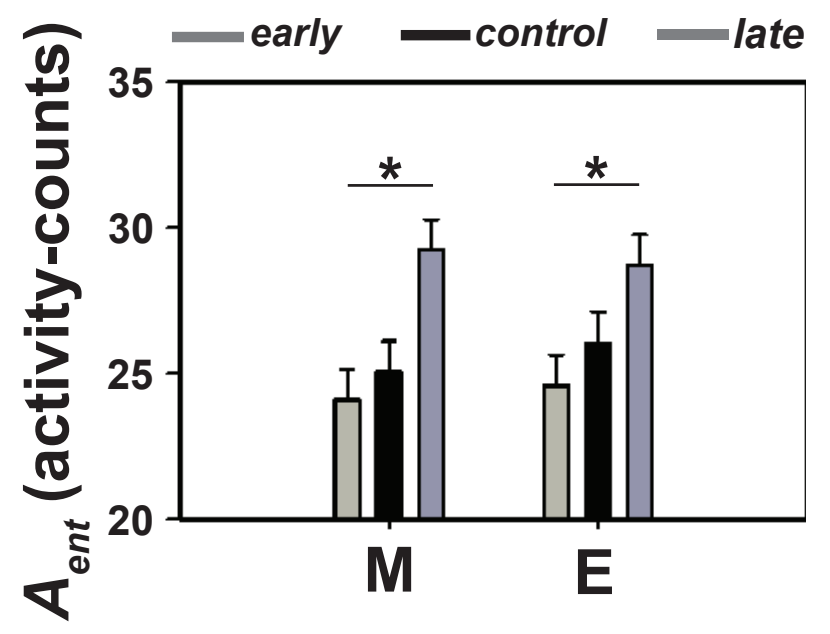

Supplementary Figure S3: Amplitude of entrainment $\left(A_{\text {ent }}\right)$ estimated as the sum of activity counts around the morning $(\mathrm{M})$ and evening $(\mathrm{E})$ peaks averaged across 7 days for all three sets of populations under LD12:12 with $4 \times 10^{-4} \mathrm{Wm}^{-2}$ light intensity during the day. The late populations exhibited significantly higher $A_{\text {ent }}$ for both $\mathrm{M}$ and $E$ peaks as compared to the other populations while the $M$ and $E$ peaks did not differ significantly from each other for a given population. Asterisks indicate statistically significant differences $(p<0.05)$. 

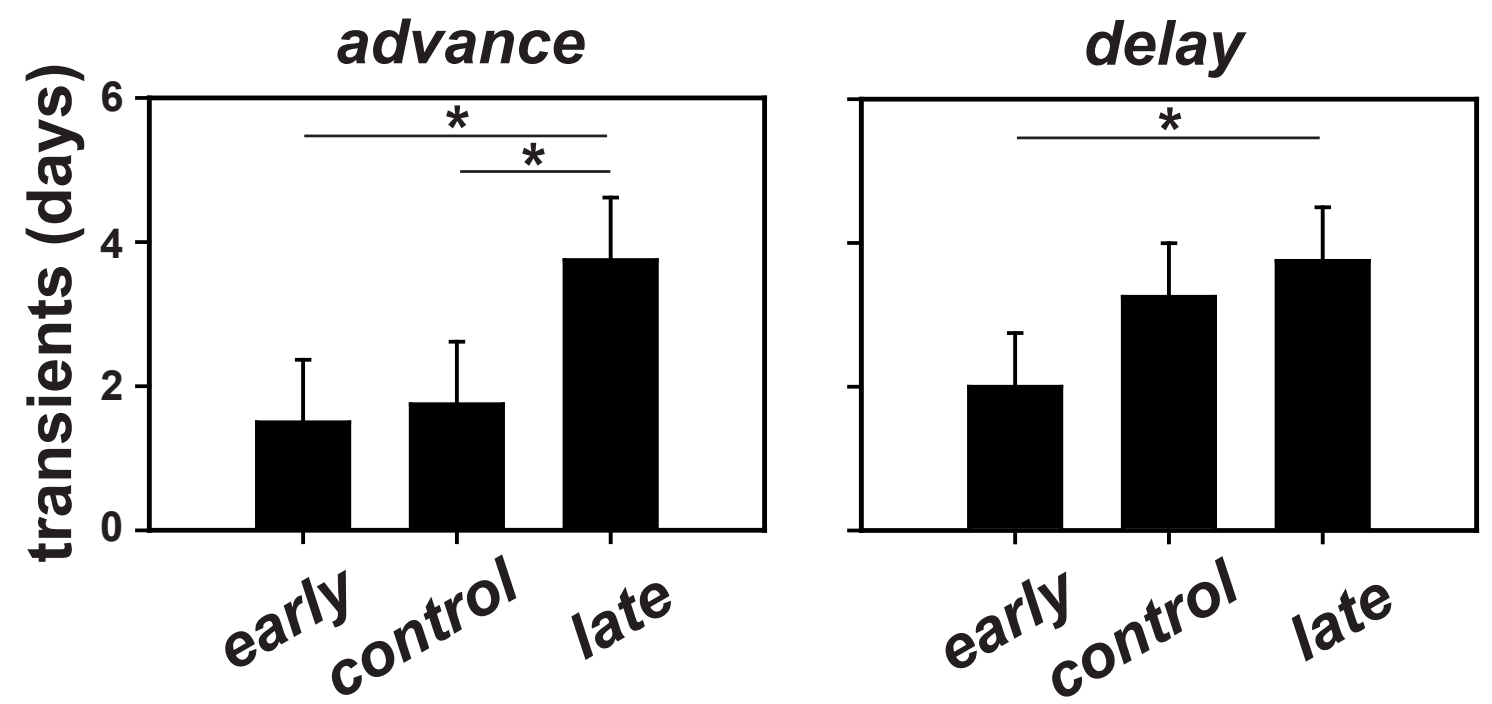

Supplementary Figure S4: Number of days (transients) required for the three sets of populations to re-entrain to 9-h phase-advance and 9-h phase-delay in light/ dark12:12 cycles. ANOVA on transients revealed a significant effect of population for both advance $\left(F_{2,6}=9.52, \mathrm{p}<0.05\right)$ and delay $\left(F_{2,6}=6.88, \mathrm{p}<0.05\right)$. In both cases the late populations took significantly longer to re-entrain as compared to the early populations while it significantly differed from the control for phase-advance and not phase-delay. Asterisks indicate statistically significant differences $(p<0.05)$. 


$\begin{array}{lll}\mathrm{R}^{2} & 0.955317674 \\ \mathrm{a} & -8.11602602 \\ \mathrm{~d} & \mathrm{~b} & 9.851402678 \\ \mathrm{c} & -3.98625742 \\ \mathrm{~d} & 1.502223689 \\ \mathrm{e} & -0.0548885\end{array}$

$\begin{array}{ll}\mathrm{R}^{2} & 0.837365931 \\ \text { a } & 9.163518506 \\ \text { b } & -4.38186548 \\ \text { c } & 0.122330037 \\ \text { d } & -0.01997385 \\ \text { e } & -6.46107614\end{array}$

$y=a+b x^{2}+c x^{4}+d x^{6}+e x^{8}+f x^{10}$

$R^{2} \quad 0.977749981$

$-1.43497958$

0.620452214

$-0.09867596$

0.004373099

$-6.61 \mathrm{E}-05$

2.61E-07

$y=a+b x+c x^{2}+d x^{3}+e x^{4}+f x^{5}$

$\begin{array}{lll} & R^{2} & 0.999950352 \\ & a & 2.418326646 \\ \mathbb{J} & b & -4.43362575 \\ \text { d } & c & 2.382178875 \\ \text { d } & -0.49683413 \\ \text { e } & 0.022850698 \\ \text { f } & 0.00162637\end{array}$

\section{$y=a+b x+c x^{2}+d x^{3}+e x^{4}+f x^{5}$}

$\begin{array}{ll}R^{2} & 0.987934691 \\ \text { a } & 11.32164822 \\ \text { b } & -24.689396 \\ \text { c } & 18.65207431 \\ \text { d } & -6.30150412 \\ \text { e } & 0.94927054 \\ \text { f } & -0.05178496\end{array}$

$y=a+b x+c x^{2}+d x^{3}+e x^{4}+f x^{5}$

$\begin{array}{ll}R^{2} & 0.96120615 \\ \text { a } & -12.9238362 \\ \text { b } & 17.8179283 \\ \text { c } & -9.84501063 \\ \text { d } & 2.738486331 \\ \text { e } & -0.38102054 \\ \text { f } & 0.020441504\end{array}$

$\begin{array}{ll}R^{2} & 0.999277944 \\ \text { a } & -2.38160908 \\ \mathrm{~b} & 1.373449817 \\ \mathrm{c} & -0.24467596 \\ \mathrm{~d} & 0.014092844 \\ \mathrm{e} & -0.000321 \\ \mathrm{f} & 2.53 \mathrm{E}-06\end{array}$

$y=a+b x+c x^{2.5}+d e^{x}+e / x^{2}$

$R^{2} \quad 0.983689312$

a $\quad 14.55127251$

b $\quad-7.10829061$

c $\quad 0.390503898$

d $\quad-0.01385338$

e $\quad-7.73199016$

$y=a+b x^{2}+c x^{4}+d x^{6}+e x^{8}$

$R^{2} \quad 0.905906231$

a $\quad 0.722655957$

b $\quad-0.45664792$

c $\quad 0.035403632$

d $\quad-0.00123564$

e 1.43E-05

$\begin{array}{ll}R^{2} & 0.936109756 \\ \text { a } & 2.351191599 \\ \text { b } & -3.42333506 \\ \text { c } & 0.113796245 \\ \text { d } & -0.017344 \\ \text { e } & 16.31920159\end{array}$

$y=a+b x+c x^{2 \ln x}+d x^{3}+e e^{x}$

$\begin{array}{ll}R^{2} & 0.975602135 \\ \text { a } & -1.99351764 \\ \text { b } & 1.050497192 \\ \text { c } & -1.71092602 \\ \text { d } & 0.54198498 \\ \text { e } & -0.02615298\end{array}$

$y=a+b x+c x^{3}+d(\ln x)^{2}+e / x^{2}$

$\begin{array}{ll}\mathrm{R}^{2} & 0.998725481 \\ \text { a } & 232.197012 \\ \text { b } & -153.013914 \\ \text { c } & 0.394368112 \\ \text { d } & 186.5907559 \\ \text { e } & -7.83 E+01\end{array}$

Supplementary Table S1: Details of polynomials obtained by curve fitting to experimental PRC data. The selection of polynomials was based on highest $R^{2}$ values indicating goodness of fit and least number of coefficients. 